\title{
Coastal Fog Tower - Design and Fabrication Process of a Vertical Fog Capture Device
}

\section{SIGRADI2018 TECHNOPOLITICAS \\ xxii congresso da sociedade iberoamericana de gráfica digital 22th conference of the iberoamerican society of digital graphics 07|08|09|novembro|2018 iau usp | são carlos | sp br}

\author{
Alberto Fernández González \\ Architect and Academic U. de Chile - FORMS| Chile | alfernan@uchile.cl \\ Susana Ortega Gómez \\ Industrial Designer U. Mayor - FORMS | Chile | su.ortega@gmail.com
}

\begin{abstract}
The coastal fog-harvesting tower project is a proposal that seeks to develop a water capture system from the condensation of coastal fog and rain, in a vertical structure that allows greater efficiency in collecting ambient humidity versus current bi-dimensional horizontal systems. The water availability in our country has declined over the last decade, so innovative solutions are required to take advantage of our unique coastal water potential. Using digital and analogue design technologies has been possible to develop a highly replicable and adaptable structural solution that can bring an affordable answer to this problem.
\end{abstract}

Keywords: Fog-Tower; water, 3d-print, digital-fabrication

\section{INTRODUCCIÓN}

El proyecto Coastal Fog-harvesting Tower es una propuesta que busca desarrollar un sistema de captura de agua a partir de la condensación de niebla costera y Iluvia, en una estructura vertical que permita una mayor eficiencia de captura de agua versus los actuales sistemas horizontales que interfieren el flujo de neblina de manera tangencial. La disponibilidad de agua en nuestro país ha disminuido durante la última década, por lo que se requieren soluciones innovadoras que aprovechen el potencial hídrico no convencional de nuestra costa.
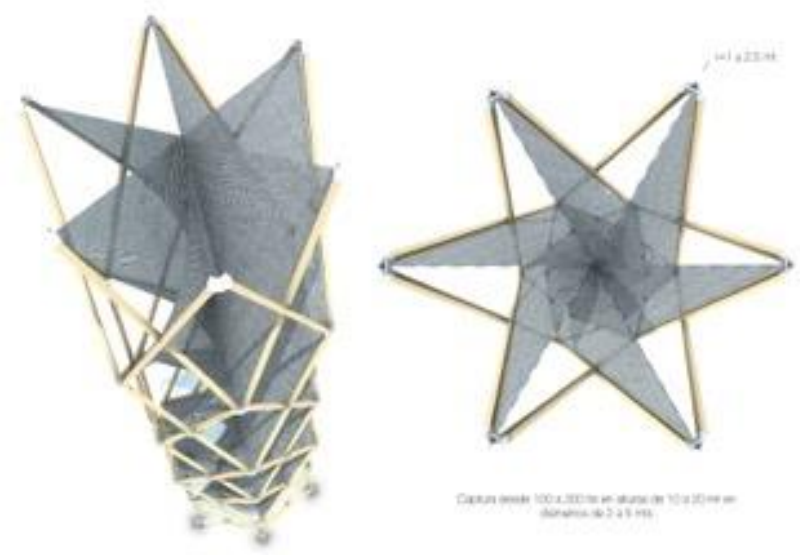

Figura 1: Sistema de captación de niebla vertical mediante una estructura generada a partir de 30 conectores impresos en 3D y largos definidos por algoritmo visual. Source: authors.
Por ello, se desarrolló en el año 2008 una propuesta conceptual para la recolección de niebla vertical, que ha evolucionado durante los años hasta la última versión (figura 1) desarrollada con el apoyo del gobierno de Chile y el programa de lucha contra la desertificación de las Naciones Unidas. El proyecto en síntesis recoge la nube de "Camanchaca", una niebla costera que se origina en el anticiclón del Pacífico de manera vertical versus los sistemas tradicionales, así como también puede optimizar la capturar de lluvia durante la época de lluvias.

\section{OBJETIVOS DE LA PROPUESTA}

Objetivo General: Desarrollar un sistema de captura vertical en base a torres de altura media (10 a $20 \mathrm{mts}$ ) que capturen agua para uso humano desde la niebla costera existente, así como de la optimización de la captura de agua de lluvia en la mayor parte del litoral centro y norte de nuestro país con alrededor de dos mil kilómetros de territorio.

Objetivos Específicos:

-Innovar en el desarrollo de estructuras verticales autoportantes y extensibles que permitan la captura de agua en base a mallas existentes en el mercado, simplificando el traslado y montaje de estas, entendiendo las realidades geográficas de nuestro litoral en términos de accesibilidad. 
-Experimentar con el uso de la madera como material estructural ligero predominante en trabajo integrado con elementos de unión fabricados mediante impresión 3d y corte laser que permitan una aproximación contemporánea tridimensional de organizaciones formales y de diseño compatibles con la captura de agua en vertical (figura 2).

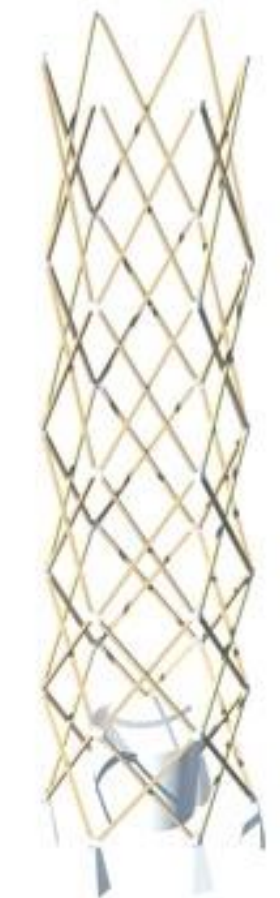

Estructura phogable en forma $X$

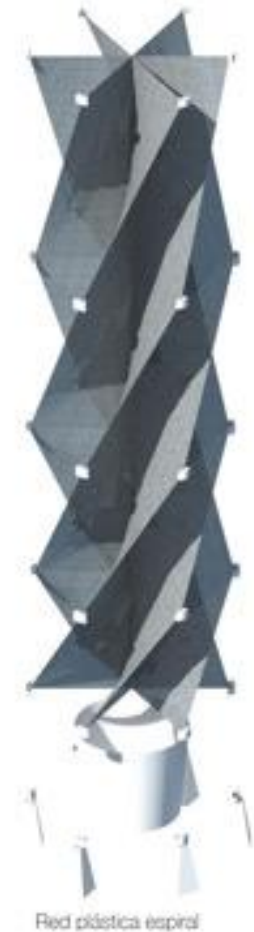

Figura 2: Sistema Estructural en base a $X$ de madera dimensionada mediante algoritmo visual (grasshopper) y malla interna en disposición espiral. Source: authors.

\section{METODOLOGÍA}

La metodología empleada se basa en 2 etapas propias de la investigación proyectual:

1.-La primera etapa de carácter teórico busca investigar los patrones de diseño estructurales tridimensionales que permitan el mínimo uso de piezas y el máximo tamaño y resistencia posible en vertical. El proceso busca documentar las características y cualidades de estos modelos mediante el análisis de piezas 3d impresas y mediante modelos virtuales, para posteriormente clasificarlos en función de las potencialidades latentes (figura 3).

2.-La segunda etapa de carácter experimental actualmente en ejecución busca poner a prueba las estructuras seleccionadas de la etapa 1 mediante modelos a escala real que definirán el o los modelos compatibles con los objetivos de esta investigación para llegar a su fabricación en serie (figura 4).

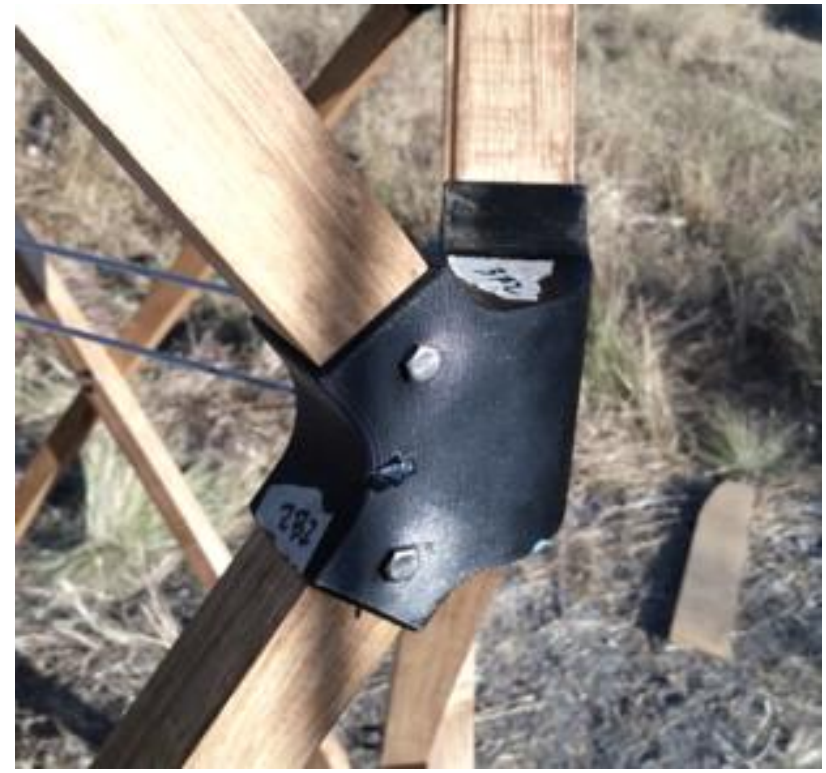

Figura 3: conector proveniente de modelo simulado, impreso en ABS para su posición definitiva en el prototipo realizado el año 2016 con el fin de probar su resistencia en el tiempo.

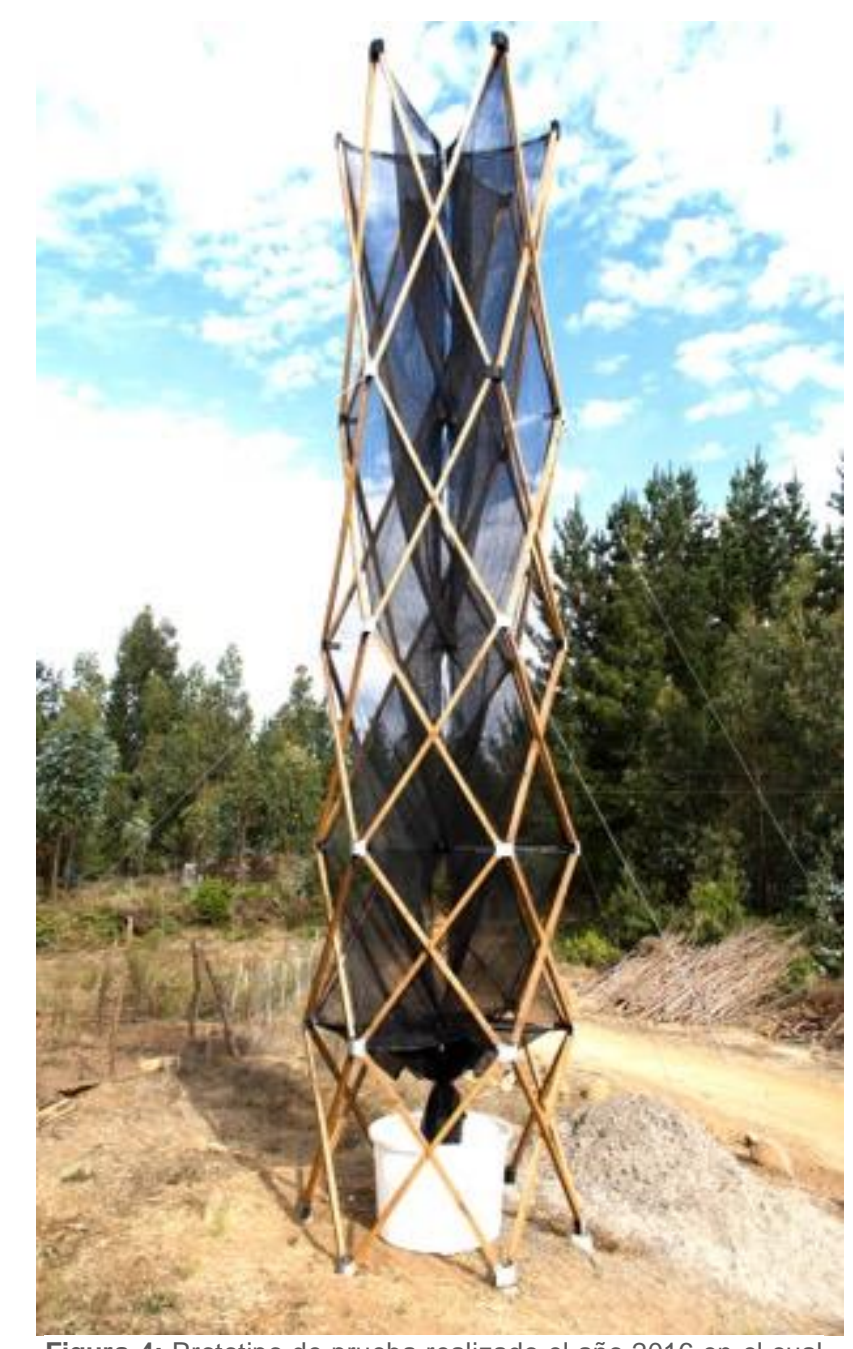

Figura 4: Prototipo de prueba realizado el año 2016 en el cual se prueba su factibilidad real, resistencia material y posibilidades de fabricación en serie. Source: authors. 


\section{EVOLUCIÓN DE LA PROPUESTA}

Siendo premiada originalmente la idea conceptual con el primer lugar en el Holcim Award 2008 para arquitectos menores de 35 años y el 3er lugar en el Evolo 2008 de New York para estructuras verticales, el proyecto comienza con una exploración formal a partir de software de modelamiento libre ( $3 d$ max) el cual permitió realizar una aproximación visual de la idea base, la cual ha evolucionando en el tiempo, desde un sistema de mega escala, a una propuesta hoy en día que busca replicarse como sistema unifamiliar o de carácter comunitario.(figura $5)$.

Actualmente se cuenta con un modelo estructural en sitio instalado desde el 2010 en los cerros aledaños a la Serena (sector cercano al aeropuerto) con el fin de testear la resistencia de este al embate del tiempo, dando hasta la fecha una estructura en base a madera tradicional que ha durado 5 años sin mantención versus las estructuras tradicionales que fracasan normalmente por problemas de esta índole en el tiempo. Este modelo sirve de base para la versión 2015-2016 la que toma lo aprendido del modelo anterior, sobretodo en lo referido al proceso de conexión y uso de material, optimizando en un $50 \%$ el peso efectivo del modelo (de 200 a $100 \mathrm{~kg}$ en su última versión). (figura 6)

Adicionalmente se cuentan con modelos virtuales de fabricación escalables a otros tamaños. el proyecto fue presentado a concursos internacionales de arquitectura y sostenibilidad y desde Diciembre de 2015 se esta testando una nueva versión de casi 10 metros de altura en el sector de Navidad en la Sexta región para ayudar a las familias aisladas en esta zona que no tienen la posibilidad de obtener agua por otro medio, con el fin de plantear la escalabilidad del proyecto hacia la maximización de captura de agua en sectores remotos de chile (figura 7).

\section{CARACTERISTICAS DEL PROYECTO}

Existen estudios preliminares realizados por varias fuentes que avalan la teoría de captura de agua en vertical a partir de niebla costera. El referente más importante en este sentido es el realizado por el climatólogo alemán Jochem Kummerov, en los cerros de Fray Jorge a mediados de los años 50 como profesor de física de la Universidad de Chile. Este modelo es uno de los pocos realizados en disposición vertical a partir de un cilindro de 2 metros de alto con un diámetro de $70 \mathrm{~cm}$. el cual fue tejido pacientemente con 1.380 hilos de perlón de $1 / 2 \mathrm{~mm}$ de espesor, condensando la niebla en diminutas gotas que se precipitan a un recipiente en su base. Otras experiencias han sido realizadas por el profesor Carlos Espinosa de la UCN en Antofagasta durante los años 60 utilizando modelos verticales y triangulados en tres dimensiones. Otros casos han sido investigados desde los años 80 por la Geógrafa Pilar Cereceda de la PUC en Alto Patache mediante sistemas principalmente mediante estructuras bidimensionales.

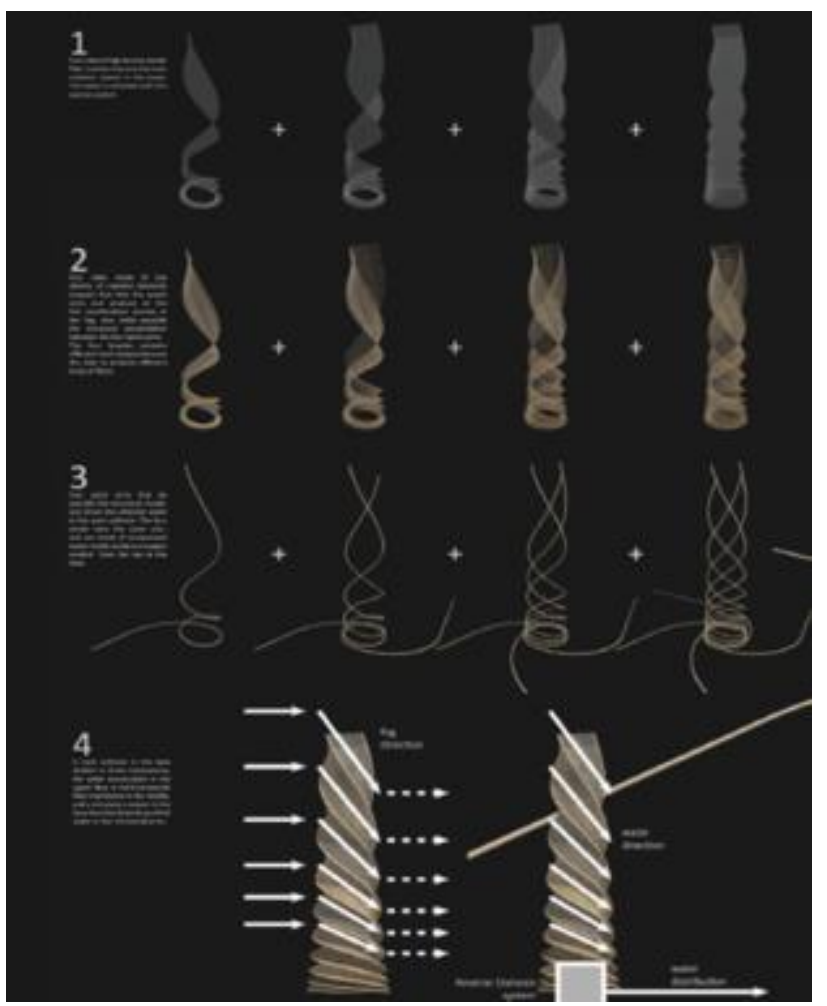

Figura 5: Etapa inicial de diseño realizado en modelador de forma libre 3dmax. Source: authors.
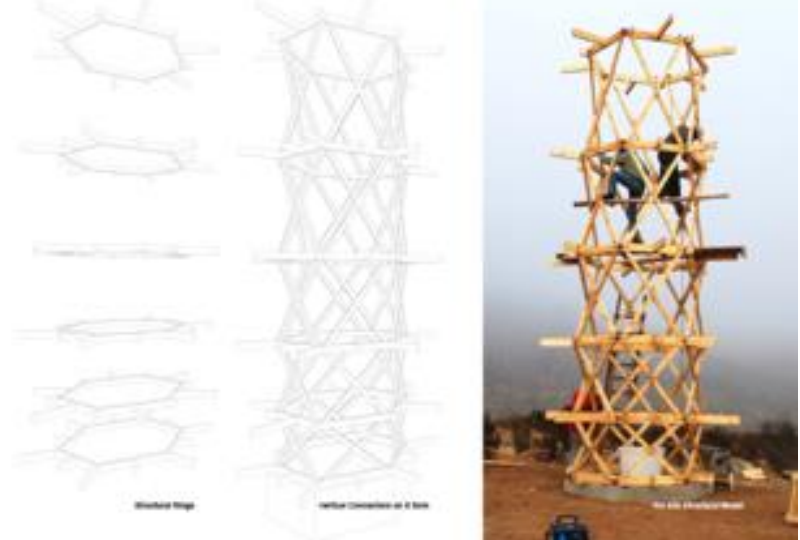

Figura 6: Modelo versión 2010 desde Rhino - Archicad con un peso final de aproximadamente 200 ks y 4 días de montaje

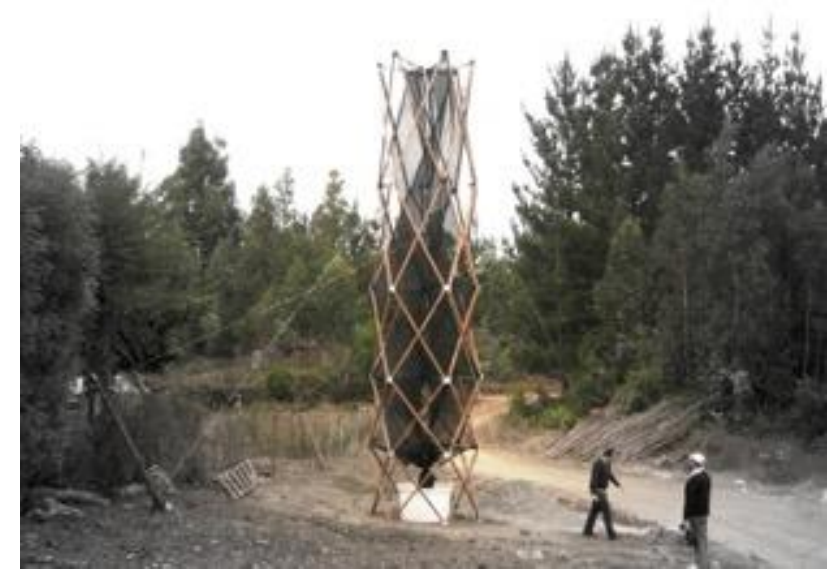

Figura 7: Modelo versión 2015-2016 desde Rhino grasshopper con un peso final de aproximadamente $100 \mathrm{ks}$ y 2 días de montaje. Source: authors. 
Los estudios demuestran la estabilidad de densidad de partículas de agua por sobre los 2 metros de altura, siendo que muchos atrapanieblas tradicionales son dispuestos en el rango de 2 a 6 metros de altura, perdiendo el potencial de la capa superior de nube a la cual no pueden acceder. Este sistema permite alcanzar alturas de hasta 20mts expandiendo el territorio de captura de agua. Por otro lado también el proyecto en si es un foco claro de expansión la captura de agua en sectores costeros aplicado a cultivos de bajo consumo hídrico con fines agrícolas tecnificados, cuya aplicabilidad es en al menos 21 países y lugares como Chile, Perú, Ecuador, Hawai, Namibia, Islas Canarias, Cabo Verde y África del sur.

\section{EFICIENCIA}

Actualmente los sistemas existentes tienen un 20\% de eficiencia de captura versus este sistema que actúa por capas helicoidales de captura que permiten adaptabilidad a los cambios de corriente de aire en comparación a un sistema plano bi-dimensional tradicional. Ya en si los costos de un sistema normal dan una rentabilidad a favor de $34 \%$ versus camiones aljibes para abastecer a zonas remotas con un valor de m2 de US\$ 7.88 dólares.

Dentro de los estudios de atrapanieblas existen 3 puntos clave para el desarrollo de estos sistemas (Pilar Cereceda - Pedro Hernández • Jorge Leiva • Juan de Dios Rivera 2015):

1.- La detección de lugares potenciales aptos para colocar atrapanieblas

2.- Mejorar el diseño estructural de estos

3.- Crear mallas más eficientes.

De los anteriores puntos, esta propuesta apunta en su proceso a optimizar el diseño estructural de los modelos, mediante la incorporación de tecnologías de fabricación digital, permitiendo la democratización del proceso productivo y dando transferibilidad al diseño a partir de la inclusión de modelamiento en base a algoritmos visuales dentro de rhino-grasshopper, pudiendo generar a partir de una versión prototipo, diversas variaciones que le dan la posibilidad de adaptarse a territorios complejos (figura 8).

Al desarrollar una estrategia hoy en día de carácter "DIY" es posible crear en un futuro cercano estrategias de masificación de estas estructuras al compartir un "algoritmo" con salida de fabricación de conectores impresos en ABS 3d, que conectan piezas estándar del mercado como son maderas de sección $2 \times 2$ presentes en el mercado global.

Hoy en día hemos creado una propuesta capaz de soportar por años los embates del tiempo, permitiendo el reemplazo en su interior de distintos tipos de malla y su estructura lo hace particularmente resistente al viento $y$ su posterior mantención lo cual abre un territorio potencial de exploración diferenciado claramente de su competencia.

\section{RESULTADOS A LA FECHA Y PASOS FUTUROS DE LA INVESTIGACIÓN}

-Rendimiento de captura de agua por m2 de suelo: La posibilidad de replicar modelos de actuar vertical desde 10 - 20 metros de altura con un diámetro de 2 - 4 metros y que puede atrapar las partículas de agua de la niebla y precipitaciones con acumulación de agua en la base de la torre dentro de un tanque puede producir un produciendo un promedio de 100 - 300 litros por día desde 3m2 de superficie de suelo versus los sistemas lineales tradicionales de captura de 6x4 metros lineales (figura 9).

-Tecnología de fácil replicación (reducción de costos): La torre fue diseñada como una espiral tridimensional de captura con una base estructural de madera desplegada, conectores reforzados $3 \mathrm{~d}$ impresos por laser CNC los cuales trabajan con un conjunto de malla plástica espiral dentro de la estructura con un valor de $\$ 1.500 .000$.- para una torre de 10 metros de altura.

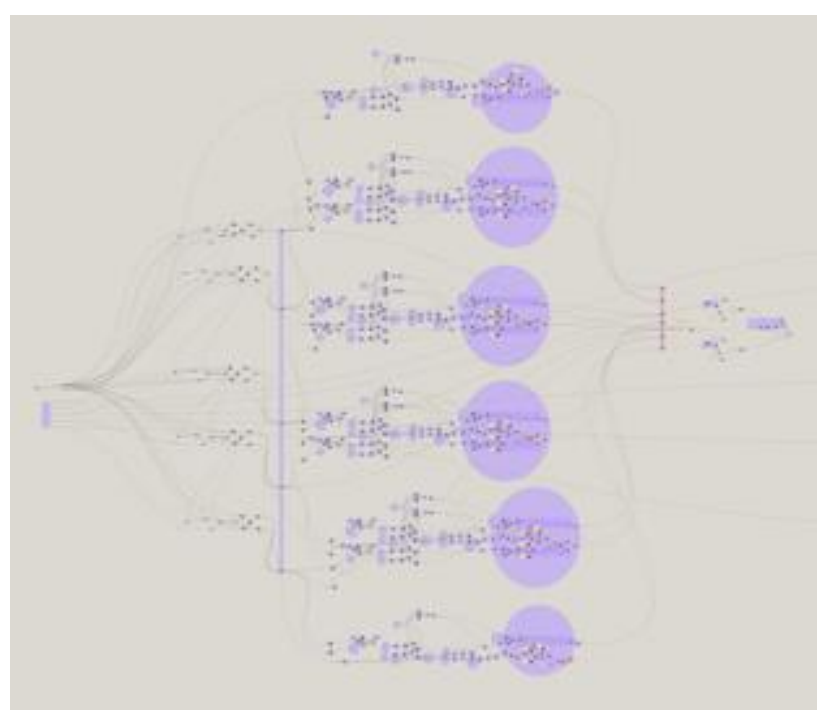

Figura 8: Definición de rhino-grasshoper que genera el modelo versión 2015-2016. Source: authors.

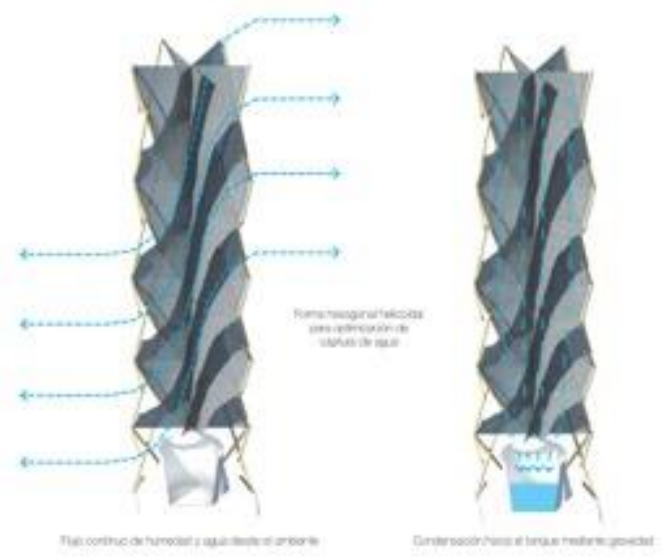

Figura 9: Esquema de funcionamiento del sistema. Source: authors.

-Condicionantes energéticas: El agua capturada en el sistema sólo utiliza viento y la gravedad como su principal mecanismo de captura. desarrollando una solución simple escalable que proporcionará agua a las comunidades rurales de Costa chilenas.

-Transportabilidad: el sistema tiene el potencial de ser plegado para una mayor facilidad de transporte y disminución de trabajo en terreno,2 tardes v/s 1 semana de trabajo con el sistema tradicional (costo de mano de obra x día y transporte). 
Por otra parte los resultados a la fecha nos demuestran que es posible agregar nuevas materiales de captura dentro del modelo estructural probado, por lo que estamos trabajando en propuestas de modelos de captura mediante estructura secundaria tri-dimensional desarrollada partir de algoritmos visuales de sub división que generan como resultado formas capaces de capturar agua sin necesidad de incluir una malla Raschel cuya duración promedio es 2 años, luego de lo cual hay que reemplazarlas, afectando la durabilidad del sistema y factibilidad en el tiempo (figura 10).

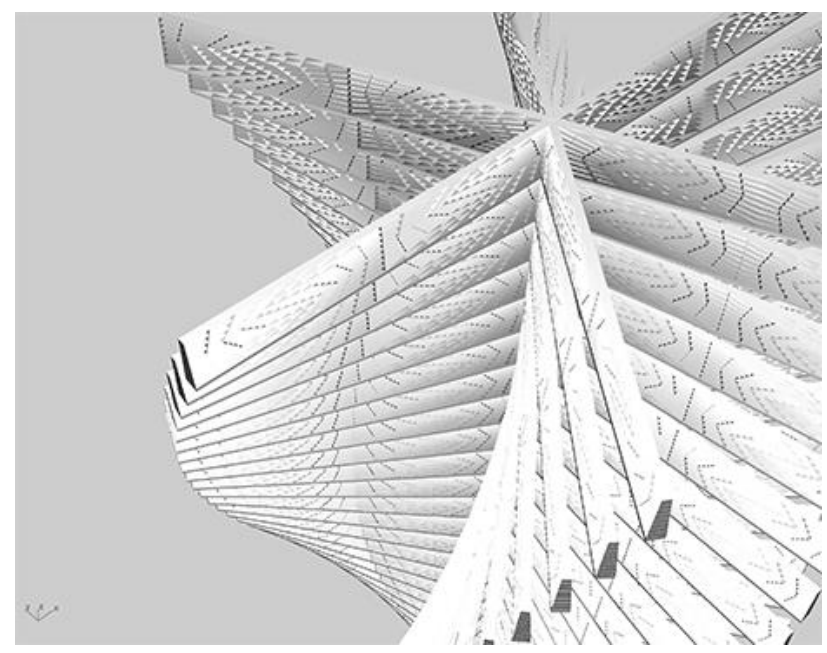

Figura 10: Modelo de subdivisión de estructura secundaria de captura mediante rhino - grasshopper para impresión 3d. Source: authors.

\section{AGRADECIMENTOS}

Damos las gracias a todas las personas e instituciones que han colaborado con nosotros en el desarrollo de esta iniciativa, en especial a Lafrge Holcim foundation (Suiza), Science Museum London (Inglaterra), ONU, Ministerio del Interior (Chile), Comcult (Alemania) y a nuestras familias que han sido parte de cada etapa realizada.

\section{REFERENCIAS}

- Cereceda, Pilar • Hernández, Pedro • Leiva, Jorge • Rivera, Juan de Dios, Agua de Niebla, 2015

- Corfo, Desarrollo y pruebas de prototipos de atrapanieblas, 2014.

- Onu, Captación y Almacenamiento de Agua de Lluvia, Opciones técnicas para la agricultura familiar en América Latina y el Caribe, 2013.

- Soto, Guido, Corporación Nacional Forestal IV Región, Captación de Agua de Las Nieblas Costeras (Camanchaca), Chile, 2000. 